\title{
CONTROL DE Sitophilus zeamais Motschulsky CON POLVOS DE FOLLAJE DE DOS ESPECIES DEL GÉNERO Eucalyptus
}

\section{CONTROL OF Sitophilus zeamais Motschulsky WITH FOLIAGE POWDERS OF TWO SPECIES OF Eucalypus GENUS}

\author{
Cristobal Higueras ${ }^{1 \mathrm{a}}$, Gonzalo Silva-Aguayo ${ }^{1 \mathrm{b*}}$, Angélica Urbina ${ }^{1 \mathrm{c}}$, Inés Figueroa ${ }^{1 \mathrm{~d}}{ }$, J. Concepción \\ Rodríguez-Maciel ${ }^{2 a}$, Angel Lagunes-Tejeda ${ }^{2 b}$, Marcela Rodríguez ${ }^{3}$ y Liliana Aguilar-Marcelino ${ }^{4}$
}

${ }^{1 a}$ Facultad de Agronomía, Universidad de Concepción, Vicente Méndez 595, Chillán, Chile

${ }^{1 b}$ Facultad de Agronomía, Universidad de Concepción, Vicente Méndez 595, Chillán, Chile https://orcid.org/0000-0002-2463-5540

${ }^{1 c}$ Facultad de Agronomía, Universidad de Concepción, Vicente Méndez 595, Chillán, Chile https://orcid. org/0000-0001-7144-5558

${ }^{1 d}$ Facultad de Agronomía, Universidad de Concepción, Vicente Méndez 595, Chillán, Chile https://orcid. org/0000-0002-9720-9710

2a Programa de Entomología y Acarología, Colegio de Postgraduados, Km 36,5 Carretera Federal, México-Texcoco, Montecillo, Estado de México, México https://orcid.org/0000-0002-1018-2229

${ }^{2 b}$ Programa de Entomología y Acarología, Colegio de Postgraduados, Km 36,5 Carretera Federal, México-Texcoco, Montecillo, Estado de México, México https://orcid.org/0000-0001-9974-7881

${ }^{3}$ Facultad de Ciencias Naturales y Oceanográficas, Universidad de Concepción, Barrio Universitario, Concepción, Chile https://orcid.org/0000-0002-2431-3701

${ }^{4}$ Centro Nacional de Investigación Disciplinaria en Salud Animal e Inocuidad, INIFAP, Km 11 Carretera Federal Cuernavaca-Cuautla, Jiutepec, Morelos, México https://orcid.org/0000-0002-8944-5430

* Autor para correspondencia E-mail: gosilva@udec.cl

\section{RESUMEN}

Los insectos plaga de los cereales almacenados causan pérdidas entre 10 y $20 \%$, lo que hace necesaria la búsqueda de alternativas de control efectivas, amigables con el medio ambiente y que no representen riegos para la salud de las personas. El objetivo de la presente investigación fue evaluar, en condiciones de laboratorio, la acción insecticida e insectistática del polvo de follaje de Eucalyptus globulus y Eucalyptus nitens para el control de Sitophilus zeamais. Las variables estudiadas fueron toxicidad por contacto y fumigación y actividad repelente y anti-alimentaria. La toxicidad por contacto y fumigación no superó el $40 \%$ de mortalidad en ninguna de las concentraciones evaluadas, aunque el tratamiento de $8,0 \%$ afectó la progenie, registrando un $80,9 \%$ de inhibición de la emergencia de insectos adultos $\left(\mathrm{F}_{1}\right)$ para E. globulus y de $86,7 \%$ en E. nitens. La totalidad de los tratamientos mostró efecto repelente y el polvo de E. nitens presentó un índice de disuasión de la alimentación (IDA) de $60,5 \%$. Se concluye que el polvo de follaje de E. globulus y E.nitens presenta potencial como repelente e inhibidor del ciclo de $S$. zeamais.

Palabras clave: Gorgojo del maíz, insecticidas vegetales, granos almacenados. 


\section{ABSTRACT}

Insect pests of stored cereals cause losses between 10 and $20 \%$, which requires the search for effective and environmentally friendly control alternatives that pose no threat to human health. The objective of this study was to evaluate under laboratory conditions the insecticidal and insectistatic activity of Eucalyptus globulus and Eucalyptus nitens foliage powder against Sitophilus zeamais. The studied variables were contact and fumigant toxicity and repellent and anti-feed activity. The contact and fumigant toxicity did not exceed $40 \%$ mortality in any of the concentrations evaluated, although the concentration of $8.0 \%$ affected the progeny, recording $80.9 \%$ inhibition of adult insect emergence $\left(\mathrm{F}_{1}\right)$ in E. globulus and $86.7 \%$ inhibition in E. nitens. All the treatments showed repellency and $E$. nitens recorded a feeding deterrence index (FDI) of $60.5 \%$. It can be concluded that E. globulus and E.nitens powder has potential as a repellent and inhibitor of the life cycle of S. zeamais.

Key words: Maize weevil, botanical insecticides, stored grains.

\section{INTRODUCCIÓN}

El almacenaje de los cereales es una de las etapas críticas del proceso productivo y se ha convertido en una práctica de elevado nivel técnico (Hagstrum y Athanassiou, 2019). El almacenamiento deficiente de granos constituye una fuente de alimento para los insectos que causan pérdidas superiores al $10 \%$ durante la producción y de 10 a $20 \%$ en poscosecha (Trivedi et al., 2018).

El gorgojo del maíz (Sitophilus zeamais Motschulsky; Coleoptera: Curculionidae), es una de las plagas de mayor relevancia de los cereales almacenados a nivel mundial (Mossi et al., 2011). Es un insecto cosmopolita y que cuenta con reportes de resistencia a los insecticidas más utilizados en su control como organofosforados (Braga et al., 2011; Ugurlu y Konus et al., 2015), piretroides (Haddi et al., 2018; Araújo et al., 2011) y fosfuro de aluminio (Daglish et al., 2014; Agriafioti et al., 2019).

En la actualidad existen múltiples opciones para combatir las plagas de insectos asociadas a granos almacenados, siendo los fumigantes sintéticos los más utilizados. Sin embargo, estos compuestos son tóxicos para mamíferos e inducen el desarrollo de resistencia en las plagas, lo cual obliga a buscar nuevas alternativas que presenten resultados similares a los convencionales pero que a la vez estén libres de los peligros que estos acarrean (Campos et al., 2019). Recientemente, ha surgido un creciente interés en la investigación sobre el uso de derivados de plantas como alternativa a los insecticidas sintéticos. Diferentes tipos de formulaciones de plantas, como polvos, extractos y aceites esenciales, están siendo investigadas por su potencial efecto insecticida de contacto y fumigante e insectistático como repelente o antialimentario (Kambouzia et al., 2009).

La mayoría de las plantas con actividad insecticida/insectistática se caracterizan por ser aromáticas y tener uso medicinal, industrial o culinario, debido a la presencia de compuestos químicos pertenecientes al metabolismo secundario de la planta y mayoritariamente del grupo de los terpenos (Isman, 2020). Algunos de estos compuestos han mostrado propiedades tóxicas contra insectos fitófagos, por lo que han sido considerados como potenciales bioplaguicidas. Uno de los metabolitos secundarios más destacado es 1,8-cineol o Eucaliptol, el cual ha registrado actividad tóxica contra varias especies de insectos de importancia médica y agrícola (Sukontason et al., 2004) y según Lee et al. (2001) es uno de los fumigantes naturales con mayor futuro.

Existen variadas referencias sobre el uso de polvos, extractos o aceites esenciales de diferentes especies del género Eucalyptus para el control de plagas de los cereales almacenados. Sin embargo, según Trivedi et al. (2018) la forma más sencilla es secar las hojas, molerlas y mezclarlas con los granos. En relación a la actividad insecticida del polvo de diferentes especies de Eucalyptus, Onuminya et al. (2018) con una concentración de 0,1g de polvo de Eucalyptus camaldulensis en 500 g de maíz, obtuvieron un $80 \%$ de mortalidad de S. zeamais; Emeka et al. (2020) también con polvo de E. camaldulensis a una concentración de 5,0\% reportaron un $65 \%$ de $S$. zeamais muertos a las 96 h de exposición. A su vez, Sitoe et al. (2020) señalan que el polvo de Eucalyptus citriodora, solo y en mezcla con ceniza, reduce significativamente el daño del maíz producido por Sitophilus oryzae. Por último, Dufera et al. (2019) indican que el polvo de Eucalyptus globulus disminuye en un $50 \%$ el daño por insectos en maíz almacenado sin afectar la germinación de las semillas.

En Chile, actualmente existen cerca de 342.000 hectáreas de plantaciones de Eucalyptus, que se cosechan para madera pulpable con edades entre 10 y 12 años. Algunas especies, como E. globulus, tienen la característica de brotar vigorosamente, produciendo biomasa en cantidades considerables, que debe ser manejada con podas o raleos (Pérez et al., 2020), generándose 
abundante material vegetal, sin mayores costos asociados, para uso industrial (Carey et al., 2006). El presente trabajo tuvo como objetivo evaluar, en condiciones de laboratorio, la acción insecticida e insectistática del polvo de follaje de E. globulus y E. nitens, para el control de S. zeamais.

\section{MATERIALES Y MÉTODOS}

El estudio se realizó en el Laboratorio de Entomología de la Facultad de Agronomía de la Universidad de Concepción, Campus Chillán, Región de Nuble, Chile.

\section{Material vegetal}

La colecta de hojas de ambas especies de Eucalyptus se realizó en un bosque de 22 años de edad ubicado en la Estación Experimental

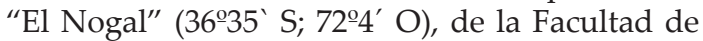
Agronomía de la Universidad de Concepción, Campus Chillán, Chile. La identificación de las especies la realizó la botánica de la Facultad de Agronomía, que además fue quien estableció los árboles en 1994. El criterio de colecta fue el de Vogel et al. (1997), que consiste en seleccionar hojas al azar, de los cuatro puntos cardinales y tanto de la periferia como del centro del árbol. Las hojas se secaron en una estufa de convección forzada (Memmert Gmbh, UNB 500, Schwabach, Germany) a $40 \pm 2{ }^{\circ} \mathrm{C}$ por 48 horas. Luego de deshidratado, el follaje se pulverizó con un molino eléctrico para granos de café (Moulinex, A5052HF, Aleçon, France) y posteriormente se tamizó con un tamiz de 18 mesch (Dual Manufacturing, Chicago, Illinois, USA), para homogeneizar su granulometría.

\section{Insectos}

Se utilizaron adultos de $S$. zeamais provenientes de colonias permanentes del Laboratorio de Entomología que se reproducen en condiciones controladas de $25 \pm 2{ }^{\circ} \mathrm{C}$ de temperatura, $60 \%$ de humedad relativa y oscuridad total, en una cámara bioclimática (Memmert Gmbh, IPS 749, Schwabach, Germany) y maíz (Zea mays L.) como sustrato alimenticio. Los insectos utilizados en los bioensayos no tenían más de siete días de edad, por lo que una semana antes de realizar los bioensayos se retiraron todos los insectos adultos de una colonia y se esperó la emergencia de la $\mathrm{F}_{1}$. En los bioensayos donde fue necesario determinar el sexo de los insectos se utilizó el criterio de Halstead (1963), quien señala que el macho posee un rostrum de menor longitud, pero mayor grosor y ornamentación que el de la hembra.

El maíz de las colonias y bioensayos se obtuvo de la Estación Experimental "El Nogal" de la Facultad de Agronomía de la Universidad de
Concepción y correspondió al cultivar Dekalb DK440 (Anasac Chile S.A). El grano se lavó con agua potable para eliminar algún residuo de insecticida y luego se sometió a condiciones de $-4,0 \pm 1^{\circ} \mathrm{C}$ de temperatura durante 72 horas, con el objetivo de eliminar alguna posible infestación previa con insectos que pudieran interferir en los resultados de los bioensayos.

\section{Actividad insecticida por contacto}

El bioensayo se realizó con la metodología de Silva-Aguayo et al. (2005), que evalúa la mortalidad, emergencia de insectos adultos $\left(\mathrm{F}_{1}\right)$ y pérdida de peso y germinación del maíz. En frascos de vidrio de $400 \mathrm{~mL}$ se depositaron 200 g de maíz, los cuales se mezclaron con el polvo de E. nitens o E. globulus en concentraciones de 0,$25 ; 0,5 ; 1,0$; 2,0; 4,0 u 8,0 \% (p/p). A continuación, cada frasco se infestó con 10 parejas de insectos y se evaluó la mortalidad a las $24,48,72$ horas y a los 7 y 15 días después de la infestación (DDI). El porcentaje de mortalidad se corrigió con la fórmula de Abbott (1925). Al finalizar la evaluación de los 15 DDI se retiraron los insectos y polvo restantes y los frascos se devolvieron a la cámara bioclimática por 40 días más (55 DDI). Completado este plazo se evaluó la $\mathrm{F}_{1}$ y la pérdida de peso del grano por diferencia entre el peso inicial (100 g) y final del maíz. Cabe destacar que para esta última variable, al inicio del bioensayo, se dejaron en la cámara bioclimática cinco frascos con $200 \mathrm{~g}$ de maíz libres de insectos y polvo de modo de registrar una posible ganancia o pérdida de peso del grano que se pudiera atribuir equivocadamente a los tratamientos o insectos. Luego de evaluada la pérdida de peso del grano, el maíz se sometió a una prueba de germinación para determinar si el polvo de follaje de E. globulus o E. nitens afectaba el poder germinativo de las semillas. En placas Petri de $9 \mathrm{~cm}$ de diámetro, acondicionadas en su base con papel filtro Advantec $\mathrm{N}^{\circ} 2$ (Advantec MFS, Inc. Pleasanton, California, USA) humedecido con agua destilada, se pusieron a germinar, a temperatura ambiente (20 $\pm 5^{\circ} \mathrm{C}$ ), 20 semillas por repetición seleccionadas de aquellas que aparentemente no presentaban daño y al cabo de una semana se calculó el porcentaje de germinación, considerando el testigo como $100 \%$. Cada tratamiento tuvo diez repeticiones que se almacenaron en una cámara bioclimática en condiciones de $25 \pm 2^{\circ} \mathrm{C}$ de temperatura, $60 \%$ de humedad relativa y oscuridad total.

\section{Actividad insecticida por fumigación}

En este bioensayo se utilizaron envases plásticos de $200 \mathrm{~mL}$, en cuyo interior se encontraba un tubo de PVC de 2,5 cm de diámetro y $5,0 \mathrm{~cm}$ de alto (SilvaAguayo et al., 2005). En el espacio comprendido entre la pared externa del tubo e interior del envase 
plástico se depositaron $20 \mathrm{~g}$ de maíz infestado con 20 insectos adultos sin diferenciación de sexo. A su vez, en el interior del tubo se ubicaron los tratamientos en concentraciones de 0,$25 ; 0,5 ; 1,0$; 2,0; 4,0 u 8,0 \% (p/p) de polvo de la especie de Eucalyptus a evaluar. Los tubos se cubrieron con una tela de tul para evitar que los insectos entraran en contacto con el polvo, pero sin evitar que los compuestos volátiles se liberasen al medio. A los 5 DDI se evaluó la mortalidad, que se corrigió con la fórmula de Abbott (1925). Cada tratamiento tuvo 10 repeticiones y durante el bioensayo, los frascos se almacenaron en una cámara bioclimática en las mismas condiciones que el bioensayo anterior.

\section{Repelencia y antixenosis}

El efecto repelente se evaluó utilizando una arena de libre elección, consistente en cinco placas Petri plásticas de $5 \mathrm{~cm}$ de diámetro, distribuidas cuatro de ellas alrededor de una placa central, formando una " $\mathrm{X}$ " y conectadas con tubos plásticos de $10 \mathrm{~cm}$ de largo y $0,25 \mathrm{~cm}$ de diámetro (Procópio et al., 2003). En las cuatro placas periféricas, se depositaron $20 \mathrm{~g}$ de maíz, pero en solo dos de ellas, opuestas diagonalmente, se aplicaron los tratamientos de polvo de E. nitens o E. globulus en concentraciones de 0,$25 ; 0,5 ; 1,0 ; 2,0$; 4,0 u $8,0 \%(\mathrm{p} / \mathrm{p})$. En la placa central se liberaron 50 insectos adultos sin diferenciación de sexo y a las 24 horas se contabilizó el número de insectos por placa. Cada arena de selección constituyó una repetición y se montaron 10 de ellas y con los valores obtenidos se calculó el índice de repelencia de Mazzonetto y Vendramim (2003) complementado con la escala de Bustos et al. (2017).

La antixenosis (no preferencia) se evaluó con la metodología de Cruzat et al. (2009) que consiste en una arena de selección, formada por ocho placas Petri plásticas de $5 \mathrm{~cm}$ de diámetro distribuidas alrededor de una placa central y conectadas a esta por tubos plásticos de $10 \mathrm{~cm}$ de largo y 0,25 cm de diámetro. En las ocho placas periféricas se depositaron $20 \mathrm{~g}$ de maíz, con los respectivos tratamientos; $0 ; 0,125 ; 0,25 ; 0,5 ; 1,0$; 2,$0 ; 4,0$ u $8,0 \%(\mathrm{p} / \mathrm{p})$ de polvo de Eucalyptus y en la placa central se liberaron 100 insectos adultos, sin diferenciación de sexo. A los 5 DDI se contabilizó el número de insectos por placa y cada arena de selección constituyó una repetición montándose 10 de ellas.

\section{Efecto anti-alimentario}

Se utilizó una modificación de la metodología de Napoleao et al. (2013), que consistió en elaborar discos con una mezcla de harina de trigo y polvo de E. nitens o E. globulus en las diferentes concentraciones a evaluar. Los discos se realizaron con una solución de $30 \mathrm{~mL}$ de agua destilada y $10 \mathrm{~g}$ de la mezcla de harina de trigo más el polvo de Eucalyptus en concentraciones de 0,$25 ; 0,5 ; 1,0 ; 2,0 ; 4,0$ u 8,0 \% (p/p). A continuación, con una micropipeta, se depositó $2 \mathrm{~mL}$ de la solución en láminas de silicona para repostería, donde se dejaron secar por 48 horas a temperatura ambiente $\left(20 \pm 5^{\circ} \mathrm{C}\right)$. Posteriormente, los discos se ubicaron en placas Petri de $5 \mathrm{~cm}$ de diámetro, que se infestaron con cinco insectos adultos, sin diferenciación de sexo, que llevaban 24 horas sin alimentarse. Cada disco se pesó al comenzar el bioensayo y a los 10 DDI para cuantificar por diferencia de peso el consumo de los insectos. Además, por tratamiento se establecieron cinco placas Petri con los discos, pero sin insectos, para calcular una posible pérdida o ganancia de peso por humedad ambiental. Cada tratamiento tuvo 10 repeticiones y con los datos obtenidos se calculó el índice de disuasión de la alimentación de Isman et al. (1990), complementado con los criterios de Liu et al. (2007).

\section{Diseño experimental y análisis estadístico}

El diseño experimental fue completamente al azar, con un arreglo factorial de $2 \times 6$ ( 2 especies de Eucalyptus y 6 concentraciones de polvo) para los bioensayos de actividad insecticida por contacto y fumigación, actividad repelente y efecto antialimentario, y de $2 \times 7$ para el de antixenosis. Los datos obtenidos, luego de verificados los supuestos de normalidad y homocedasticidad con las pruebas de Shapiro-Wilks y Levene, se transformaron con la fórmula arcoseno $(x / 100)^{1 / 2} y$ se sometieron a un análisis de varianza (ANOVA) y a una prueba de comparación de medias de Tukey con una significancia del 5\% $(\alpha=0,05)$ con el software Statistical Analysis System (SAS).

\section{RESULTADOS Y DISCUSIÓN}

El análisis estadístico factorial mostró que las interacciones (especie de Eucalyptus $\mathrm{x}$ concentración) no fueron significativas para los resultados de los bioensayos de efecto insecticida por contacto $(\mathrm{p}=0,99)$, efecto insecticida por fumigación $(\mathrm{p}=0,96)$ y antixenosis (no preferencia) $(p=0,81)$ (Tabla 1). Lo anterior indica que no existe relación entre la especie de Eucalyptus y la efectividad del tratamiento. Por tanto, se realizó un análisis de varianza independiente para cada especie de Eucalyptus.

\section{Bioensayo de actividad insecticida por contacto}

La mortalidad de S. zeamais, obtenida con el polvo de ambas especies de Eucalyptus, fue inferior a $30 \%$ (Tabla 2). La mayor toxicidad se registró a los 15 DDI en la concentración 
Tabla 1. Significancia de la interacción en el análisis de varianza en diferentes bioensayos con polvo de dos especies de Eucalyptus (E. globulus y E. nitens) en concentraciones de 0,25; 0,5; 1,0; 2,0; 4,0 y $8,0 \%(\mathrm{p} / \mathrm{p})$ para el control de Sitophilus zeamais.

Table 1. Significance of statistical interaction in the analysis of variance in different bioassays with powder of two Eucalyptus species at concentrations of $0.25,0.5,1.0,2.0,4.0$ and $8.0 \%(\mathrm{w} / \mathrm{w})$ for the control of Sitophilus zeamais.

\begin{tabular}{lc}
\hline \multicolumn{1}{c}{ Bioensayo } & p-valor* \\
\hline Toxicidad por contacto & $0,99 \mathrm{~ns}$ \\
Emergencia de insectos adultos $\left(\mathrm{F}_{1}\right)$ & $0,16 \mathrm{~ns}$ \\
Pérdida de peso del grano & $0,92 \mathrm{~ns}$ \\
Germinación & $0,99 \mathrm{~ns}$ \\
Toxicidad por fumigación & $0,96 \mathrm{~ns}$ \\
Antixenosis & $0,81 \mathrm{~ns}$ \\
\hline
\end{tabular}

*Significancia $\mathrm{p} \leq 0,05 \quad$ ns=no significativo.

Tabla 2. Mortalidad a los 15 DDI y Emergencia $\left(\mathrm{F}_{1}\right)$ a los 55 DDI de adultos de $S$. zeamais y pérdida de peso y germinación de maíz tratado con diferentes concentraciones de polvo de follaje de Eucalyptus globulus y Eucalyptus nitens.

Table 2. Mortality at 15 DAI and Emergence $\left(\mathrm{F}_{1}\right)$ at 55 DAI of $S$. zeamais adults and weight loss and germination of treated maize with different concentrations of Eucalyptus globulus y Eucalyptus nitens powder.

\begin{tabular}{cccccc}
\hline Especie & $\begin{array}{c}\text { Concentración } \\
\mathbf{( \% )}\end{array}$ & $\begin{array}{c}\text { Mortalidad } \\
\mathbf{( \% )}\end{array}$ & $\begin{array}{c}\text { Emergencia } \\
\left(\mathbf{F}_{\mathbf{1}} \mathbf{)}(\mathbf{\%})\right.\end{array}$ & $\begin{array}{c}\text { Pérdida peso } \\
\mathbf{( \% )}\end{array}$ & $\begin{array}{c}\text { Germinación } \\
\mathbf{( \% )}\end{array}$ \\
\hline \multirow{4}{*}{ E. globulus } & 0,25 & $2,5 \mathrm{a}$ & $84,5 \mathrm{~d}$ & $39,9 \mathrm{~d}$ & $98,6 \mathrm{a}$ \\
& 0,5 & $3,8 \mathrm{a}$ & $64,4 \mathrm{c}$ & $31,8 \mathrm{~cd}$ & $98,6 \mathrm{a}$ \\
& 1,0 & $8,8 \mathrm{ab}$ & $49,9 \mathrm{bc}$ & $28,9 \mathrm{bc}$ & $100,0 \mathrm{a}$ \\
& 2,0 & $13,8 \mathrm{ab}$ & $45,3 \mathrm{~b}$ & $26,8 \mathrm{bc}$ & $97,3 \mathrm{a}$ \\
& 4,0 & $18,8 \mathrm{~b}$ & $34,9 \mathrm{~b}$ & $20,3 \mathrm{~b}$ & $97,3 \mathrm{a}$ \\
\hline \multirow{4}{*}{ E. nitens } & 8,0 & $28,8 \mathrm{~b}$ & $19,1 \mathrm{a}$ & $11,3 \mathrm{a}$ & $94,5 \mathrm{a}$ \\
\hline & $\mathrm{CV}(\%)$ & 9,7 & 8,8 & 8,8 & 3,5 \\
\hline & 0,25 & $2,5 \mathrm{a}$ & $87,6 \mathrm{e}$ & $41,8 \mathrm{~b}$ & $100,0 \mathrm{a}$ \\
& 0,5 & $3,8 \mathrm{ab}$ & $67,4 \mathrm{~d}$ & $38,1 \mathrm{~b}$ & $97,3 \mathrm{a}$ \\
& 1,0 & $7,5 \mathrm{ab}$ & $57,4 \mathrm{~cd}$ & $31,5 \mathrm{~b}$ & $97,3 \mathrm{a}$ \\
& 2,0 & $10,0 \mathrm{abc}$ & $46,4 \mathrm{c}$ & $29.0 \mathrm{~b}$ & $95.9 \mathrm{a}$ \\
& 4,0 & $16,3 \mathrm{bc}$ & $30,9 \mathrm{~b}$ & $24,8 \mathrm{ab}$ & $95,9 \mathrm{a}$ \\
& 8,0 & $27,5 \mathrm{c}$ & $13,3 \mathrm{a}$ & $11,9 \mathrm{c}$ & $97,3 \mathrm{a}$ \\
\hline
\end{tabular}

Tratamientos con igual letra, no difieren estadísticamente por el test de Tukey $(\alpha=0,05)$.

ANOVA se llevó a cabo con datos transformados por fórmula arcoseno $(x / 100)^{1 / 2}$

$\mathrm{CV}=$ Coeficiente de variación.

DDI = Días Después de la Infestación.

de $8,0 \%$ con 28,8 y $27,5 \%$ de insectos muertos para E. globulus y E. nitens, respectivamente. Aunque esta concentración, en ambas especies, no presentó diferencias estadísticas con 2,0 y $4,0 \%$, que no superaron el $20 \%$ de mortalidad. Esto último indica que la efectividad del polvo no aumenta significativamente al incrementar su concentración. En la evaluación a las 24,
48, 72 horas y 7 DDI la mortalidad fue inferior a $10 \%$. Considerando el criterio propuesto por Salvadores et al. (2007) quienes clasifican como prometedores aquellos tratamientos con una mortalidad mayor al $40 \%$, ninguna de las concentraciones evaluadas en este bioensayo cumple con este umbral. Silva et al. (2003) reportaron resultados similares para polvo de $E$. 
globulus, a una concentración de 2,0\%, con 1,7\% de mortalidad para S. zeamais. Igualmente, los resultados de la presente investigación coinciden con Bittner et al. (2008), quienes señalan que a pesar de que 1,8-cineol es el principal componente del aceite esencial de E. globulus, alcanzando hasta un $82,6 \%$ de su composición total, este compuesto no presenta toxicidad por contacto significativa. González-Guiñez et al. (2016) evaluaron el aceite esencial de estas mismas especies de Eucalyptus, obteniendo una toxicidad por contacto que no superó el 10\% de mortalidad, confirmando la baja actividad insecticida de contacto de E. globulus y E. nitens. En cuanto a la emergencia de insectos adultos $\left(\mathrm{F}_{1}\right)$, en la concentración más alta evaluada $(8,0 \%)$, se obtuvo $19,1 \%(80,9 \%$ de inhibición) de progenie $\left(\mathrm{F}_{1}\right)$ en E. globulus y $13,3 \%(86,7 \%$ de inhibición) en E. nitens (Tabla 2). Este tratamiento, en ambas especies, presentó diferencia significativa con las restantes concentraciones, lo que indica que el efecto inhibitorio del polvo en la $\mathrm{F}_{1}$ se incrementa significativamente al aumentar su concentración. Cabe destacar que con la concentración de 2,0\%, tanto de E. globulus como E. nitens, a pesar de que $S$. zeamais mostró una mortalidad inferior a $30 \%$, este tratamiento presentó una $\mathrm{F}_{1}$ menor a $50 \%$, lo que se podría deberse a un efecto ovicida o larvicida del polvo. Lo anterior coincide con González-Guiñez et al. (2016) para el aceite esencial de E. globulus y E. nitens contra S. zeamais y con Slimane et al. (2014) para E. globulus contra Orgya trigotephras Boisduval (Lepidoptera, Lymantriidae), concluyendo estos últimos que esta especie posee efecto larvicida, debido a su elevada concentración de 1,8-cineol. Además, Salvadores et al. (2007) plantearon como referencia de efectividad una reducción del 50\% de $\mathrm{F}_{1}$, respecto al testigo, por lo que los resultados de esta investigación permiten señalar que el polvo de Eucalyptus, en concentraciones iguales o superiores a 2,0\%, afecta el ciclo reproductivo de S. zeamais. Según González et al. (2011), lo anterior podría deberse a una interferencia con la oviposición de la hembra o la eliminación directa de los huevos. Al respecto, estudios realizados por Izakmehri et al. (2013) con aceite esencial de Eucalyptus camaldulensis, determinaron que la longevidad, fecundidad y fertilidad de la hembra de Callosobruchus maculatus se ve afectada severamente por el aceite esencial, pero no así la proporción de sexos de la progenie. Igualmente, Gehlot y Singhvi (2006) y Singh (2011) reportan una reducción significativa de la oviposición de C. maculatus como efecto de un extracto de hojas de E. globulus. Izakmehri et al. (2013), en bioensayos con semillas impregnadas con aceite esencial y expuestas a hembras de C. maculatus, concluyeron que el aceite no impide ni afecta la tasa de oviposición. Además, estudios histológicos, realizados por Bindhu et al. (2017), señalan que, a pesar de que algunos extractos o aceites esenciales afectan significativamente el número de oviposturas por hembra, no se observan daños en las diferentes estructuras de los ovarios, por lo que la reducción de la $\mathrm{F}_{1}$ se podría atribuir a una muerte del huevo o menores posturas por falta de alimentación y no a un efecto esterilizante en la hembra.

La pérdida de peso del grano en todos los tratamientos con polvo de Eucalyptus fue significativamente menor respecto al testigo que se consideró como $100 \%$. En la concentración de $8,0 \%$, se obtuvo una pérdida de peso de $11,3 \%$ en E. globulus y $11,9 \%$ para $E$. nitens versus un $58,9 \%$ del testigo (Tabla 2). La concentración de 8,0\% no presentó diferencias estadísticas con $4,0 \%$ en E. nitens, pero sí en E. globulus, lo que permite indicar que en esta última especie el efecto podría incrementarse significativamente al aumentar la concentración de polvo. Sin embargo, estos valores son menores a los de Silva et al. (2003), quienes con 2,0\% de concentración de polvo de $E$. globulus obtuvieron un 5,5\% de pérdida de peso, mientras que en la presente investigación con la misma concentración de polvo se obtuvo 26,8 y $29 \%$, para E. globulus y E. nitens, respectivamente. La divergencia con los resultados de Silva et al. (2003) podría deberse a una diferencia en la concentración de los compuestos químicos presentes en el polvo, lo que provocaría un mayor efecto repelente o disuasivo de la alimentación, produciendo un menor consumo por parte de los insectos.

La germinación del maíz no se vio afectada significativamente por ninguno de los tratamientos con polvo de Eucalyptus, ya que todos lograron una germinación superior al $90 \%$ y no presentaron diferencias estadísticas entre ellos (Tabla 2). Este porcentaje de germinación permite calificar las semillas como aptas para su uso comercial, según los umbrales establecidos por el Servicio Agrícola y Ganadero (SAG) de Chile, que, en la resolución N 379 de 1991, señala que la semilla corriente debe lograr como mínimo un $85 \%$ de germinación, y la semilla certificada un 90\% (Cerda et al., 2000). De esta forma, se puede señalar que el polvo de E. globulus y E. nitens no es fitotóxico para las semillas y no interfiere con el proceso germinativo del grano, por lo que podría utilizarse tanto para proteger cereales destinados a alimentación como para semilla. Lo anterior es una ventaja para el polvo de Eucalyptus, debido a que en investigaciones con maíz tratado con polvo de otras especies vegetales como $P$. boldus (Silva et al., 2003; Pérez et al., 2007), C. zeylanicum y P. nigrum (Salvadores et al., 2007), se observaron porcentajes de germinación inferiores al 80\%. 


\section{Actividad insecticida por fumigación}

Ninguno de los tratamientos evaluados superó el $40 \%$ de mortalidad. La mayor toxicidad se obtuvo con la concentración de $8,0 \%$, con 22,5 y $20 \%$ de mortalidad, con E. globulus y E. nitens, respectivamente (Tabla 3). Sin embargo, este tratamiento no presentó diferencia estadística con las concentraciones de 0,$5 ; 1,0,2,0$ y $4,0 \%$, indicando que un aumento de la concentración del polvo no implica necesariamente una mayor mortalidad. En el caso del aceite esencial de estas dos especies del género Eucalyptus, GonzálezGuiñez et al. (2016) reportaron valores de mortalidad superiores al $70 \%$ en los tratamientos de 20, 25, 30, $35 \mu \mathrm{L}$ aceite esencial $\mathrm{L}^{-1}$ aire con $E$. nitens y con $35 \mu \mathrm{L}$ aceite esencial $\mathrm{L}^{-1}$ aire en $E$. globulus, lo que indicaría que el polvo posee una menor concentración de compuestos volátiles con efecto insecticida que el aceite esencial. Según Alzogaray et al. (2100), existe una correlación positiva entre la actividad fumigante y la concentración de 1,8-cineol y $\alpha$-pineno. Igualmente, Sukontason et al. (2004) atribuyen el efecto fumigante de Eucalyptus, en general, a 1,8-cineol, quienes evaluaron este compuesto con un $98 \%$ de pureza contra mosca doméstica (Musca domestica: Diptera: Muscidae) y la mosca de las letrinas (Chrysomya megacephala; Diptera: Calliphoridae), obteniendo, en ambas especies, un alto efecto fumigante y repelente. Finalmente, Lee et al. (2001), también evaluaron 1,8-cineol puro contra $S$. oryzae, Tribolium castaneum y Rhyzopertha dominica, a una concentración de $20 \%$, obteniendo un $95 \%$ de mortalidad, por lo que el $8,0 \%$ de polvo evaluado en el presente estudio se puede considerar como bajo, lo que explicaría la reducida mortalidad obtenida con este tratamiento.

\section{Repelencia y antixenosis}

El índice de repelencia en todos los tratamientos fue menor 1, lo cual, de acuerdo a Mazzonetto y Vendramim (2003), clasifica el polvo de ambas especies de Eucalyptus como repelente, destacándose que, incluso, con la concentración más baja $(0,25 \%)$, tanto en E. globulus como $E$. nitens, se registró repelencia (Tabla 4); aunque, de acuerdo a la escala de intensidad de la repelencia de Bustos et al. (2017) el efecto de 0,25\% se clasifica como repelencia débil. Sin embargo, a partir de la concentración de $0,5 \%$, el polvo de $E$. nitens presenta una repelencia alta $(0,5)$ a muy alta $(1,0 ; 2,0$ y $4,0 \%)$ mientras que en E. globulus 0,5 y $1,0 \%$ mostraron repelencia alta, y 2,0\% repelencia muy alta. En el caso del aceite esencial de E. globulus y E. nitens, González-Guiñez et al. (2016) también obtuvieron repelencia alta y muy alta en todos los tratamientos evaluados, lo que indicaría que, aunque los compuestos fumigantes estén en menor concentración en el polvo, esta es suficiente para provocar un comportamiento disuasivo en el insecto. Los resultados obtenidos también coinciden con lo reportado para polvos de otras especies como Ch. ambrosioides (SilvaAguayo et al., 2005), C. annuum var. grossum, Cuminum cyminum L. (Apiaceae) y Origanum vulgare L. (Lamiaceae) (Salvadores et al., 2007). Aunque, algo que diferencia al polvo de

Tabla 3. Mortalidad de adultos de S. zeamais por efecto fumigante del polvo de follaje de E. globulus y E. nitens.

Table 3. Mortality of S. zeamais adults by the fumigant effect of E. globulus and E. nitens foliage powder.

\begin{tabular}{ccc}
\hline & & $\begin{array}{c}\text { Mortalidad } \\
\mathbf{( \% )}\end{array}$ \\
\hline $\begin{array}{c}\text { Concentración } \\
\mathbf{( \% )}\end{array}$ & E. globulus & E. nitens \\
\hline 0,25 & $2,5 \mathrm{a}$ & $0,0 \mathrm{a}$ \\
0,5 & $5,0 \mathrm{ab}$ & $2,5 \mathrm{ab}$ \\
1,0 & $7,5 \mathrm{ab}$ & $7,5 \mathrm{ab}$ \\
2,0 & $7,5 \mathrm{ab}$ & $10,0 \mathrm{ab}$ \\
4,0 & $17,5 \mathrm{ab}$ & $15,0 \mathrm{ab}$ \\
8,0 & $22,5 \mathrm{~b}$ & $20,0 \mathrm{~b}$ \\
$\mathrm{CV}(\%)$ & 12,1 & 13,3 \\
\hline
\end{tabular}

Tratamientos con igual letra no difieren estadísticamente por el test de Tukey $(\alpha=0,05)$.

ANOVA se llevó a cabo con datos transformados por fórmula arcoseno $(\mathrm{x} / 100)^{1 / 2}$ 
Tabla 4. Repelencia de adultos de S. zeamais en maíz tratado con polvo de follaje de E. globulus y E. nitens.

Table 4. Repellency against S. zeamais adults in treated maize with E. globulus and E. nitens foliage powder.

\begin{tabular}{ccc}
\hline & \multicolumn{2}{c}{ Índice de Repelencia (IR)* } \\
\hline $\begin{array}{c}\text { Concentración } \\
(\%)\end{array}$ & E. globulus & E. nitens \\
\hline 0,25 & 0,6 & 0,6 \\
0,5 & 0,5 & 0,2 \\
1,0 & 0,2 & 0,2 \\
2,0 & 0,2 & 0,1 \\
4,0 & 0,1 & 0,1 \\
\hline
\end{tabular}

${ }^{*} \mathrm{IR}<1$; indica que tratamiento es repelente (Mazzonetto y Vendramim, 2003).

Eucalyptus spp. con las especies anteriormente mencionadas, es su efectividad en bajas concentraciones, ya que mientras estas muestran repelencia con concentraciones superiores a $1,0 \%$, el polvo de E. globulus y E. nitens iguala o supera dichos resultados, con las concentraciones de 0,25 y $0,5 \%$. Por tanto, esto permite calificar el polvo de ambas especies de Eucalyptus como una alternativa eficaz para la prevención de ataques de $S$. zeamais.

En el bioensayo de antixenosis los insectos infestaron las placas Petri tanto tratadas como testigo. Sin embargo, la preferencia fue significativamente menor en aquellas que contenían alguno de los tratamientos de polvo de Eucalyptus (Tabla 5). En la concentración de $0,125 \%$ (la más baja evaluada), la preferencia, respecto al total de insectos, fue de 12,5 y $13,5 \%$, para E. globulus y E. nitens, mientras que el testigo mostró 45,9 y $53 \%$, respectivamente. El menor porcentaje de insectos por placa se obtuvo con la concentración de $8,0 \%$ con 2,5 y $0,5 \%$ de preferencia por parte de $S$. zeamais para E. globulus y E. nitens. Aunque, en esta última no se observaron diferencias estadísticas entre las concentraciones evaluadas, pero sí con el testigo. En E. globulus la preferencia por todas las concentraciones fue significativamente menor que el testigo $\mathrm{y}$, a su vez, las concentraciones de $0,125,0,25$ y $0,5 \%$ mostraron una preferencia estadísticamente mayor que $8,0 \%$, lo que indica una relación inversa entre preferencia y concentración de polvo de E. globulus. Estos resultados son superiores a los obtenidos con otras especies como $C$. ambrosioides, que con 1,0 y 2,0\% de concentración alcanzó 69,8 y $62,9 \%$ de preferencias, respectivamente (Silva-Aguayo et al., 2005), y a una especie de la familia Fabaceae, identificada con el código 49-1-XIV, que con 1,0\% de concentración obtuvo $42 \%$ de preferencia por parte de S. zeamais (Gónzalez et al., 2009). El hecho de que el polvo de E. globulus y E. nitens requiera de una menor concentración para lograr una menor preferencia de S. zeamais, confirma los resultados obtenidos en el bioensayo de repelencia y califica el polvo de follaje de estos árboles como una eficaz estrategia preventiva contra el ataque de $S$. zeamais.

\section{Efecto anti-alimentario}

Los mayores índices de disuasión de la alimentación (IDA) se obtuvieron, en ambas especies de Eucalyptus, con la concentración de $8,0 \%$, alcanzando $47,2 \%$ en E. globulus y $60,5 \%$ en E. nitens (Tabla 5). Los tratamientos con concentraciones de polvo iguales o inferiores a $1,0 \%$, alcanzaron un IDA menor a $20 \%$, lo que, según la escala de Liu et al. (2007), clasifica a dichos tratamientos como sin capacidad de disuasión alimentaria. Por otra parte, las concentraciones de 2,0, 4,0 y 8,0\% de E. globulus, lograron un IDA superior a $20 \%$, pero menor a 50\%, lo que, según los autores mencionados anteriormente, indica un efecto anti-alimentario débil. Finalmente, en $E$. nitens la concentración de $8,0 \%$ mostró un IDA de $60,5 \%$, que clasifica al tratamiento como con inhibición moderada. Estos resultados son similares a los obtenidos por otros autores con aceite esencial o extractos de diferentes especies de Eucalpyptus. Saedi (2014) evaluó el efecto antialimentario del aceite esencial de E. globulus y E. camaldulensis, obteniendo que $C$. maculatus consumió en menor cantidad la dieta mezclada con aceite de E. globulus que la control. Además, Hamzavi y Moharramipour (2017), determinaron que el aceite esencial de E. camaldulensis disminuyó la tasa de crecimiento y de consumo de dieta de Tribolium consufum. Por último, Ebadollahi (2011) determinaron que los volátiles del aceite 
Tabla 5. Preferencia y disuasión de la alimentación de adultos de $S$. zeamais alimentados con maíz tratado con polvo de follaje de E. globulus y E. nitens.

Table 5. Feeding preference and deterrence of $S$. zeamais adults fed with maize treated with $E$. globulus and E. nitens foliage powder.

\begin{tabular}{llcc}
\hline Especie & $\begin{array}{c}\text { Concentración } \\
\mathbf{( \% )}\end{array}$ & $\begin{array}{c}\text { Preferencia } \\
\mathbf{( \% )}\end{array}$ & $\begin{array}{c}\text { IDA } \\
\mathbf{( \% )}\end{array}$ \\
\hline & 0,0 & $45,9 \mathrm{c}$ & -- \\
& 0,25 & $12,5 \mathrm{~b}$ & $5,2 \mathrm{a}$ \\
E. globulus & 0,5 & $11,5 \mathrm{~b}$ & $11,1 \mathrm{a}$ \\
& 1,0 & $11,0 \mathrm{ab}$ & $16,2 \mathrm{a}$ \\
& 2,0 & $6,6 \mathrm{ab}$ & $20,5 \mathrm{ab}$ \\
& 4,0 & $6,0 \mathrm{ab}$ & $28,6 \mathrm{ab}$ \\
CV (\%) & 8,0 & $4,0 \mathrm{a}$ & $47,2 \mathrm{c}$ \\
\hline & & 2,5 & 12,3 \\
\hline & 0,0 & $53,0 \mathrm{~b}$ & -- \\
& 0,25 & $13,5 \mathrm{a}$ & $3,9 \mathrm{a}$ \\
& 0,5 & $10,0 \mathrm{a}$ & $13,3 \mathrm{~b}$ \\
& 1,0 & $9,5 \mathrm{a}$ & $17,8 \mathrm{~b}$ \\
& 2,0 & $7,5 \mathrm{a}$ & $24,5 \mathrm{bc}$ \\
& 4,0 & $5,0 \mathrm{a}$ & $32,2 \mathrm{c}$ \\
& 8,0 & $1,0 \mathrm{a}$ & $60,5 \mathrm{~d}$ \\
\hline
\end{tabular}

Tratamientos con igual letra, no difieren estadísticamente por el test de Tukey $(\alpha=0,05)$. ANOVA se llevó a cabo con datos transformados por fórmula arcoseno $(x / 100)^{1 / 2}$ $\mathrm{CV}=$ Coeficiente de variación.

IDA = Índice de disuasión de la alimentación.

E. globulus disminuyen la tasa de alimentación de T. castaneum.

Finalmente, la presente investigación permite aseverar que el polvo de E. globulus y E. nitens posee una prometedora actividad insectistática, con potencial para prevenir de manera eficaz los ataques de $S$. zeamais en granos almacenados. Sin embargo, es necesaria una mayor investigación del polvo de estas especies de Eucalyptus, junto con la respectiva validación en condiciones de bodega.

\section{CONCLUSIONES}

Los resultados obtenidos en la presente investigación permiten concluir que el polvo de E. globulus y E. nitens no presenta toxicidad significativa por contacto ni fumigación contra S. zeamais. Sin embargo, posee actividad insectistática como repelente y anti-alimentario, además de reducir la emergencia de insectos adultos $\left(\mathrm{F}_{1}\right) \sin$ afectar la germinación de semillas de maíz.

\section{LITERATURA CITADA}

Abbott, W.S. 1925. A method for computing the effectiveness of an insecticide. J. Econ. Entomol. 18(2):265-267.

Agrafioti, P., C.G. Athanassiou, and M.K. Nayak. 2019. Detection of phodphine resistance in major stored-product insects in Greece and evaluation of a field resistance test kit. J. Stor. Prod. Res. 82:40-47.

Alzogaray,R., A. Lucia, E. N. Zerba and H. M. Masuh. 2011. Insecticidal activity of essential oils from eleven Eucalyptus spp. and two hybrids: lethal and sublethal effects of their major components on Blattella germánica. J. Econ. Entomol. 104(2):595-600.

Araújo, R.A., M.S. Williamson, C. Bass, L.M. Field, and I. R. Duce. 2011. Pyrethroid resistance in Sitophilus zeamais is associated with a mutation (T929l) in the voltaje-gated sodium channel. Insect Mol. Biol. 20(4):437-445.

Bindhu, V.R. S. Ganga and S. Dayanandan. 2017. Effect of plant leaf extracts on fecundity in Callosobruchus chinensis L. (Coleoptera: Chrysomelidae: Bruchinae). J. Biopest. 10(2):140-145. 
Bittner, M.L., M.E. Casanueva, C.C. Arbert, M.A. Aguilera, V.J. Hernández, and J.V. Becerra. 2008. Effects of essential oils from five plant species against the granary weevils Sitophilus zeamais and Acanthoscelides obtectus (Coleoptera). J. Chilean Chem. Soc. 53(1):1455-1459.

Braga, L.S., A.S. Correa, E.J.G. Pereira, and R.N.C. Guedes. 2011. Face or flee? Fenitrotion resistance and behavioral response in populations of the maize weevil, Sitophilus zeamais. J. Stor. Prod. Res. 47:161-167

Bustos, G., G. Silva, S. Fisher, I. Figueroa, A. Urbina, y J.C. Rodríguez. 2017. Repelencia de mezclas de aceites esenciales de boldo, laurel chileno y tepa contra el gorgojo del maíz. Southwest. Entomol. 42(2):551-562.

Campos, E.R.V., P.L. Proenca, J.L. Oliveira, M. Bakshi, P.C. Abhilash, and L.F. Faceto. 2019. Use of botanical insecticides for sustainable agriculture: Future perspectives. Ecol. Indic. 105:483-495.

Carey, P., A. Figueroa, y P. Valenzuela. 2006. Evaluación técnica de un sistema tradicional de cosecha en plantaciones de Eucalyptus globulus de corta rotación en Valdivia, Chile. Bosque 27(3):272-276.

Cerda, E., G. Aparicio, G. Fernández, S. González, A. Peña, y R. Messina. 2000. Normas generales y específicas de certificación de semillas [en línea]. Servicio Agrícola y Ganadero, Chile. Disponible en https://www2.sag.gob.cl/ sag_al_dia/consultas_publicas/Proyecto_ Modificatorio_Normas_Generales20102017. pdf (Consulta 29 de abril 2021).

Cruzat, M., G. Silva, H. Serri, y R. Hepp. 2009. Protección de ocho cultivares de trigo con polvo de Peumus boldus Molina contra Sitophilus zeamais Motschulsky. Idesia 27(2):39-46.

Daglish, G.J., M.K. Nayak, and H. Pavic. 2014. Phosphine resistance in Sitophilus oryzae (L.) from eastern Australia: Inheritance, fitness and prevalence. J. Stor. Prod. Res. 59:237244.

Ebadollahi, A. 2011. Antifeedant activity of essential oils from Eucalyptus globulus Labill and Lavandula stoechas L. on Tribolium castaneum Herbst (Coleoptera: Curculionidae). Biharean Biol. 5(1):810.

Emeka, C.P.O., F.K. Ewete and S.T. Ebeniro. 2020. Efficacy of Eucalyptus leaf (Eucalyptus camaldulensis), Moringa seed (Moringa oleifera) and Pirimiphos-methyl powders against maize weevil (Sitophilus zeamais) in Stored Maize. J. Exp. Agric. Int. 42(5):85-90.
Dufera, L.T., A.Y. Tadesse, W.S. Gobena and C. G. Kuyu. 2019. On Farm evaluation of Eucalyptus globulus Labill leaf and Chenopodium ambrosioides L. whole plant powder against storage insect pests in stored maize at Sokoru district in Jimma zone of Oromia regional state, Ethiopia. Psyche. Doi: 10.1155/2019/2306742

Gehlot, L. and P.M. Singhvi. 2006. Effect of plant extracts against Callosobruchus maculatus and seed germination of moth bean (Vigna aconitifolia). J. Applied Zoo. Res. 18(1):165168.

González, S., O. Pino, R.S. Herrera, N. Valenciaga, D. Fortes, y D. Sánchez. 2009. Control de Sitophilus zeamais con polvos vegetales de una especie de la familia Fabacea (49-1-XIV). Rev. Cuba. Cienc. Agríc. 43(3):321-325.

González, S., O. Pino, R.S. Herrera, N. Valenciaga, D. Fortes, y D. Sánchez. 2011. Potencialidades de los polvos de Lonchocarpus punctatus en el control de Sitophilus zeamais. Rev. Cuba. Cienc. Agríc. 45(1):89-94.

González-Guiñez, R., G. Silva-Aguayo, A. Urbina-Parra y M. Gerding-González. 2016. Aceite esencial de Eucalyptus globulus Labill y Eucalyptus nitens H. Deane \& Maden (Myrtaceae) para el control de Sitophilus zeamais Motschulsky. Chilean J. Agric. Anim. Sci., ex Agro-Ciencia. 32(3):204-216.

Haddi, K., W.R. Valbon, L.O.V. Jumbo, L.O. de Oliveira, R.N.C. Guedes, and E.O. Oliveira. 2018. Diversity and convergence of mechanisms involved in pyrethroid resistance in the stored grain weevils, Sitophilus spp. Scientific Reports 8:16361. DOI: 10.1038/s41598-018-34513-5.

Hagstrum, D.W., and C.G. Athanassiou. 2019. Improving stored product insect pest management: theory to practice. Insects. 10(10):332. doi:10.3390/insects10100332

Halstead, D.G.H. 1963. External sex differences in stored-products Coleoptera. Bull. Entomol. Res. 54(1):119-134.

Hamzavi, F. and S. Moharramipour. 2017. Chemical composition and antifeedant activity of essential oils from Eucalyptus camaldulensis and Callistemon viminalis on Tribolium confusum. IJAT. 13(3):413-424.

Isman, M.B., O. Koul, A. Luczynski, and J. Kaminski. 1990. Insecticidal and antifeedant bioactivities of neem oils and their relantionship to azadirachtin content. J. Agric. Food Chem. 38(6):1406-1411.

Isman, M. 2020. Commercial development of plant essential oils and their constituents as active ingredients in bioinsecticides. Phytochemistry Reviews 19:235-241. 
Izakmehri, K., M. Saber, A. Mehrvar, M. B. Hassanpouraghdam and S. Vojoudi. 2013. Lethal and sublethal effects of essential oils from Eucalyptus camaldulensis and Heracleum persicum against the adults of Callosobruchus maculatus. J. Insect Sci. 13:152. Doi: 10.1673/031.013.15201.

Kambouzia, J., M. Negahban, and S. Moharramipour. 2009. Fumigant toxicity of Eucalyptus leucoxylon against stored product insects. American-Eurasian J. Sustain. Agric. 3(2):229-233.

Lee, B.H., W.S. Choi, S.E. Lee, and B.S. Park. 2001. Fumigant toxicity of essential oils and their constituent compounds towards the rice weevil, Sitophilus oryzae (L.). Crop Prot. 20(4):317-320.

Liu, Z.L., S.H. Goh, and S.H. Ho. 2007. Screening of Chinese medicinal herbs for bioactivity against Sitophilus zeamais Motschulsky and Tribolium castaneum (Herbst). J. Stored Prod. Res. 43:290296.

Mazzonetto, F., e J. Vendramim. 2003. Efeito de pós de origem vegetal sobre Acanthoscelides obtectus (Say) (Coleoptera: Bruchidae) em feijão armazenado. Neotrop. Entomol. 32(1):145-149.

Mossi, A.J., V. Astolfi, G. Kubiak, L. Lerin, C. Zanella, G. Toniazzo, et al. 2011. Insecticidal and repellency activity of essential oil of Eucalyptus sp. against Sitophilus zeamais Motschulsky (Coleoptera, Curculionidae). J. Sci. Food Agric. 91(2):273-277.

Napoleao, T.H., B.R. Belmonte, E.V. Pontual, L. Pereira, R. Araújo, L. Paiva, et al. 2013. Deleterious effects of Myracrodruon urundeuva leaf extract and lectin on the maize weevil, Sitophilus zeamais (Coleoptera, Curculionidae). J. Stored Prod. Res. 54:26-33.

Onuminya, T.O., O.O. Agboola, S.R. Ezeribe. 2018. Insecticidal evaluation of some botanical powders as stored grain protectans against Sitophilus zeamais (Motchulsky) (Coleoptera: Curculionidae); a concern for postharvest loss. Annals of West University of Timişoara-Series of Biology. 21(2):157-164.

Pérez, S., C. Renedo, A. Ortiz, and F. Ortiz. 2020. Residual biomass in Eucalyptus globulus plantations according to stand quality. Biomass \& Bioenergy 141: Doi: 10.1016/j. biombioe.2020.105699 .

Pérez, F., G. Silva, M. Tapia, and R. Hepp. 2007. Variación anual de las propiedades insecticidas de Peumus boldus sobre Sitophilus zeamais. Pesq. Agropec. Bras. 42(5):633-639.

Procópio, S., J. Vendramim, J.I. Ribeiro, e J. dos Santos. 2003. Bioatividade de diversos pós de origem vegetal em relação a Sitophilus zeamais Mots. (Coleoptera: Curculionidae). Ciênc. Agrotecnol. 27(6):1231-1236.
Saedi, K. 2014. Antifeedant and growth inhibitory activities of essential oils from Eucalyptus globulus and Eucalyptus maculatus on Callosobruchus maculatus (Coleoptera: Chrysomelidae). Plant Protection Journal. 6(4):391-400.

Salvadores, Y., G. Silva, M. Tapia, y R. Hepp. 2007. Polvos de especias aromáticas para el control del gorgojo del maíz, Sitophilus zeamais Motschulsky, en trigo almacenado. Agric. Téc. (Chile) 67(2):147-154.

Singh, R. 2011. Evaluation of some plant products for their oviposition deterrent properties against the Callosobruchus maculatus (F.) on Chick pea seeds. J. Agric. Technol. 7(5):13631367.

Silva, G., D. Pizarro, P. Casals, y M. Berti. 2003. Evaluación de plantas medicinales en polvo para el control de Sitophilus zeamais Motschulsky en maíz almacenado. Rev. Bras. Agrociênc. 9(4):383-388.

Silva-Aguayo, G., R. Kiger-Melivilu, R. HeppGallo, y M. Tapia-Vargas. 2005. Control de Sitophilus zeamais con polvos vegetales de tres especies del género Chenopodium. Pesq. Agropec. Bras. 40(10):953-960.

Sitoe, M.D., P.M. Neves and J. Zorzete. 2020. Plant derived powders as alternatives for Sitophilus oryzae L. (Coleoptera: Curculionidae) control in stored maize grains. AJFAND. 20(4):1623616248.

Slimane, B.B., O. Ezzine, S. Dhahri, and M.L.B. Jamaa. 2014. Essential oils from two Eucalyptus from Tunisia and their insecticidal action on Orgyia trigotephras (Lepidopteras, Lymantriidae). Biol. Res. 47(1):29-36.

Sukontason, K.L., N. Boonchu, K. Sukontason, and M. Choochote. 2004. Effects of Eucalyptol on house fly (Diptera: Muscidae) and bow fly (Diptera: Calliphoridae). Rev. Inst. Med. Trop. 46(2):97-101.

Trivedi, A., N. Nayaj, and J. Kumar. 2018. Recent advances and review on use botanicals from medicinal and aromatic plants in stored grain pest management. J. Entomol. Zool. Stud. 6(3):295-300.

Ugurlu, S., and M. Konus. 2015. Determination of organophosphate resistance status and mechanism in Sitophilus zeamais Motschulsky (Coleoptera: Curculionidae) from Turkish J. Biochem. 40(5):417-422.

Vogel, H., I. Razmilic, y U. Doll. 1997. Contenido de aceite esencial y alcaloides en diferentes poblaciones de boldo (Peumus boldus Mol.). Cienc. Investig. Agrar. 24(1):1-6. 